\title{
PERSPECTIVES OF SMALL BUSINESS DEVELOPMENT UNDER THE CONDITIONS OF UNCERTAINTY CAUSED BY COVID PANDEMICS
}

\author{
TEMUR SHENGELIA \\ Doctor of Economic Sciences, Professor \\ Ivane Javakhisvili Tbilisi State University, \\ Academician of the Georgian Academy of Economic Sciences, Georgia \\ shengelia.temur@gmail.com
}

Abstract. A market economic system based on the means of production is characterized by fundamental uncertainty. The differences between the fundamental uncertainties and the risks lie in the fact that it is impossible to empirically measure the level and dynamics of future threats. A correct understanding of the uncertainty is essential for the study and analysis of the crisis. Under the influence of the COVID-19 pandemic, world economy found itself in a new global crisis, which had a significant impact on the rise of economic and social uncertainty, the degree of which is so high that it is impossible to predict the model of the economic and social development due to the current pandemic. The current economic crisis is characterized not only by global losses but also by the limited sources of compensation. According to scientists (Adžić \& Al-Mansour, 2021) a crisis of this magnitude is the most unprecedented economic event of the last century.

The COVID-19 world pandemic crisis has limited a traditional opportunities for small business, accordingly has emerged a new one. The most difficult problem was created in the value chain. Small business is a heavily dependent on consumers and suppliers behaviour, therefore, changes in the value chain have a significant impact on all of its participants. The closure of national borders has led to the disruption of the logistics network;

And after the reduction of the stocks, it threatens to almost all participants in this network.

It is important to consider the uncertainties and risks that are involved while studying small business crisis behavior. As a result even the most trained institutions are approaching disaster. After a pandemic, the universe will find itself in a larger, no less turbulent zone.

Prior to the crisis, cost was the most important criterion while choosing a supply chain that had led to single suppliers and small stocks for small businesses. As it seems during the pandemic, this choice turned out to be one of the most important causes of the small business crisis. At the same time, dependence on one supplier has undergone a transformation into the need to diversify stocks, that has led to an increase in funding sources and which has led to the financial instability of a small company.

These examples of the correlation between environmental and small business threats have illustrated the need for a global vision in order to adapt anti-crisis behavior model .A global vision is certainly needed. There is no single, optimal choice for overcoming uncertainty, however, the importance of the multivariate strategies is an important. Small firms found themselves unprepared for such approaches, leading to a high level of uncertainty. As well as, when a small business is in the early stages of its life cycle, the lack of experience limits its choice, and the lack of resources prevents it from choosing an effective strategy, that has exacerbated the problems of the small firms.

KEYWORDS: FUNDAMENTAL UNCERTAINTY, CORONAVIRUS, SMALL BUSINESS, CRISIS, RESEARCH, DIGITIZATION OF BUSINESS.

For citation: Shengelia, T., (2021). Perspectives of small business development under the conditions of uncertainty caused by COVID pandemics. Globalization and Business. 12, 77-82. (In Georgian). https://doi.org/10.35945/gb.2021.12.010 


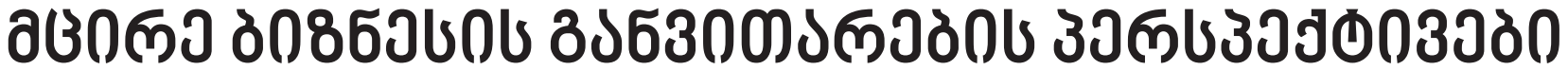

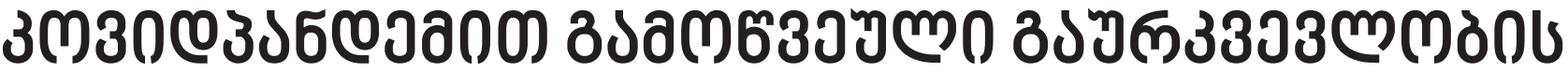

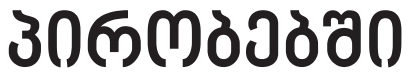

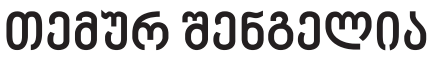

эзмбмдаз

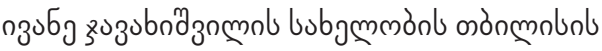

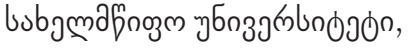

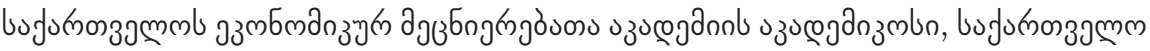

shengelia.temur@gmail.com

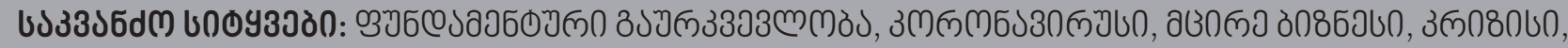

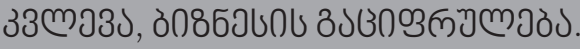

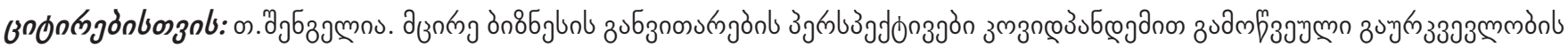

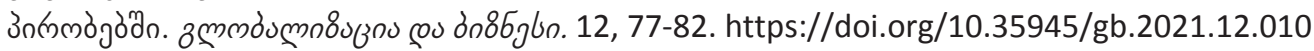

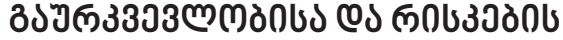

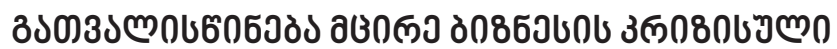

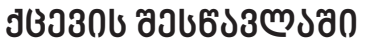

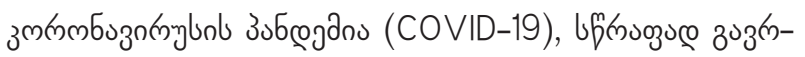

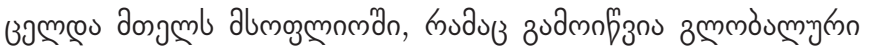

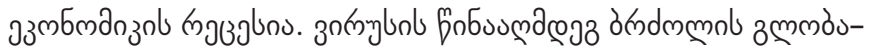

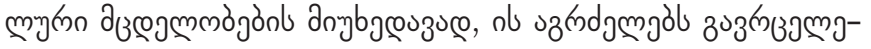

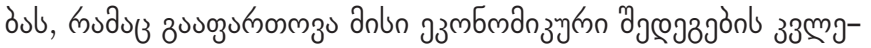

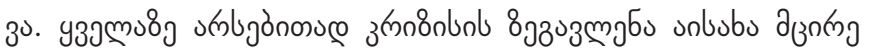

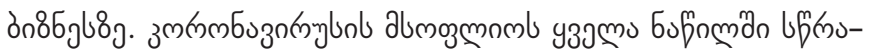

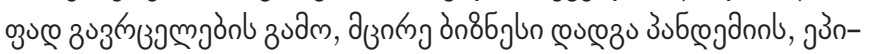

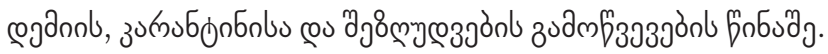

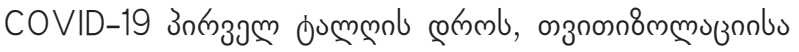

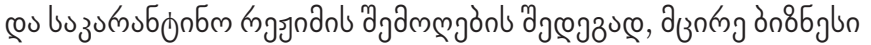

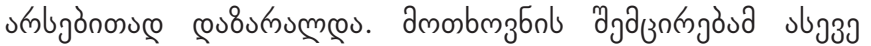

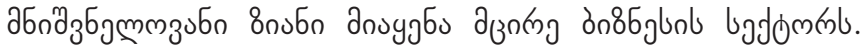

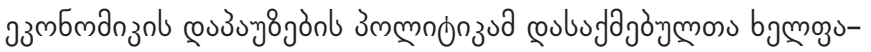

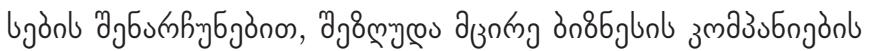
budan

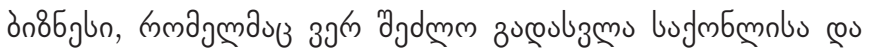

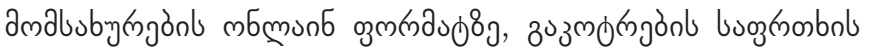

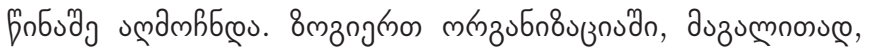

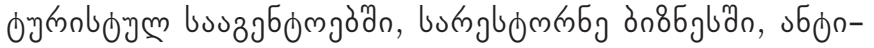

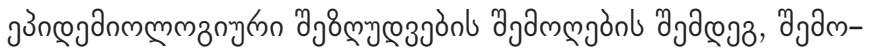

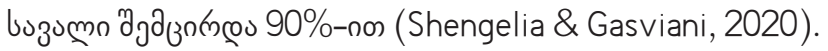

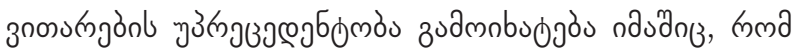

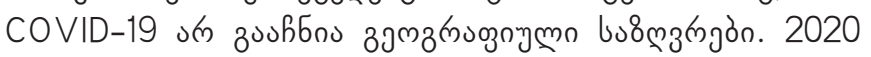

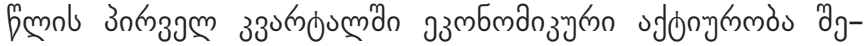

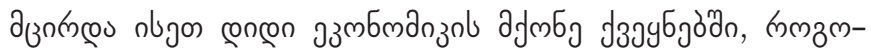

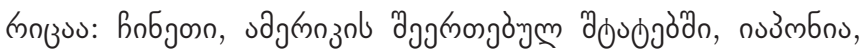

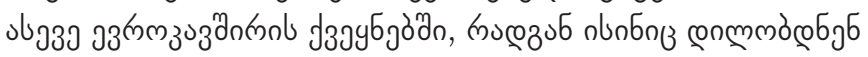

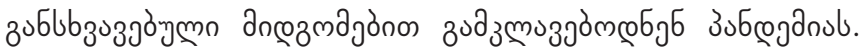

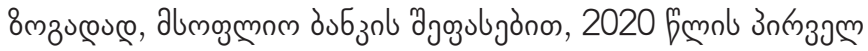

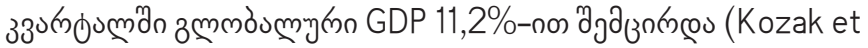

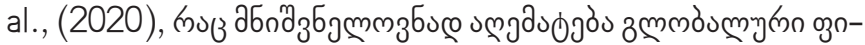

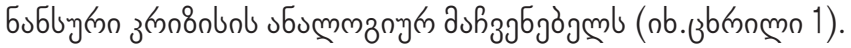

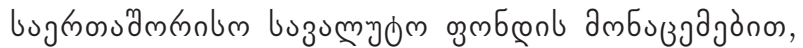

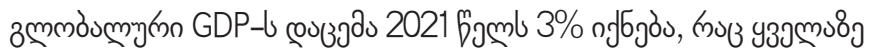

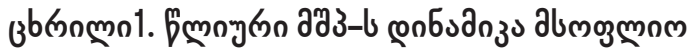

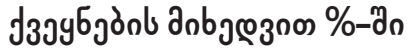

\begin{tabular}{|c|c|c|c|c|}
\hline & 2017 & 2018 & 2019 & 2020 \\
\hline alıмозмом дચЗ & 3,3 & 3,0 & 2,4 & $-7,7$ \\
\hline 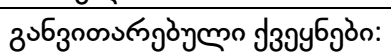 & 2,5 & 2,1 & 1,6 & $-8,4$ \\
\hline ১Әฎ & 2,4 & 2,9 & 2,3 & $-7,9$ \\
\hline эзпмзиздипо & 2,5 & 1,9 & 1,2 & $-10,1$ \\
\hline ns3m6ns & 2,2 & 0,3 & 0,7 & $-6,8$ \\
\hline 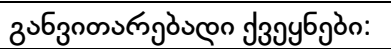 & 4,5 & 4,3 & 3,5 & $-6,6$ \\
\hline hoбgoso & 6,8 & 6,6 & 6,1 & $-4,9$ \\
\hline 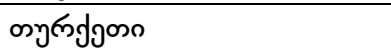 & 7,5 & 2,8 & 0,9 & $-6,8$ \\
\hline agflozo & 2,1 & 2,2 & $-0,3$ & $-8,7$ \\
\hline buycol skudgos & $-0,7$ & 2,4 & 0,3 & $-5,7$ \\
\hline "бертуов & 7,0 & 6,1 & 4,2 & $-9,0$ \\
\hline buabmgos sozmnz & 1,4 & 0,8 & 0,2 & $-8,0$ \\
\hline
\end{tabular}

Буриюn: OECD, 2020. 


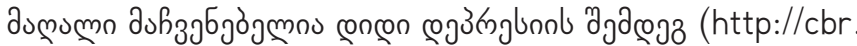
ru/Collection).

उsбе

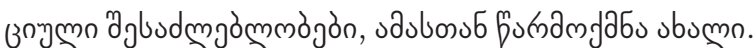

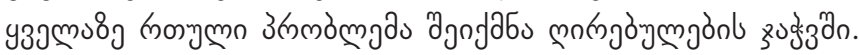

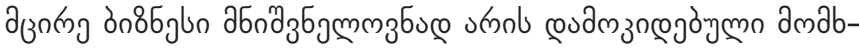

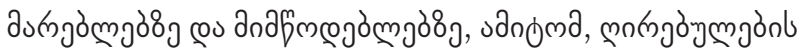

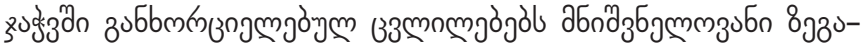

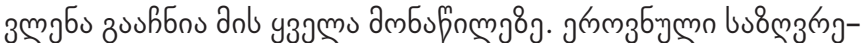

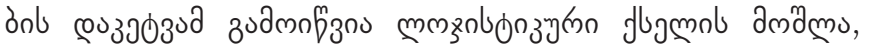

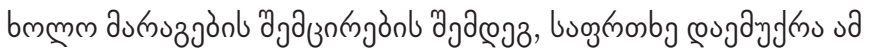
لjugmol ononfanb yzams ambufipngl (Berishvili, 2020).

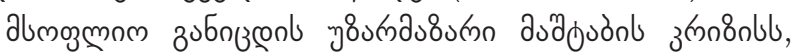

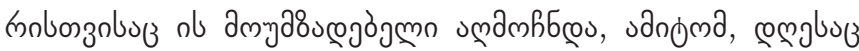

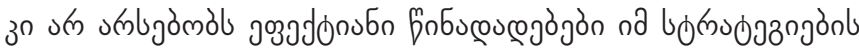

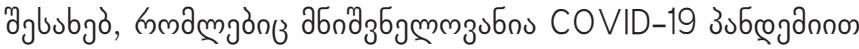

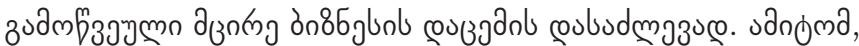

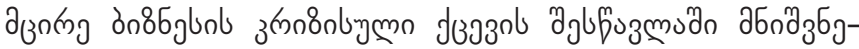

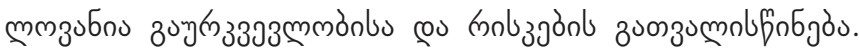

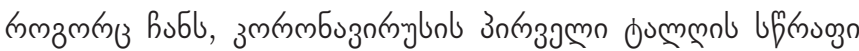

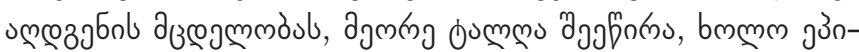

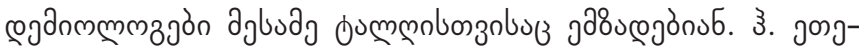

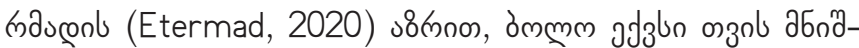

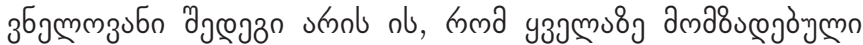

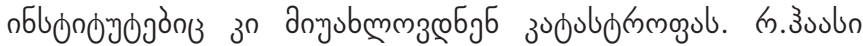

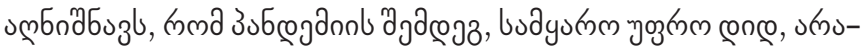

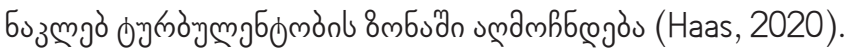

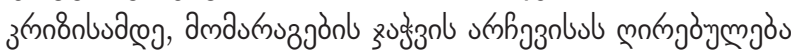

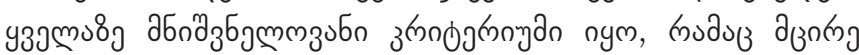

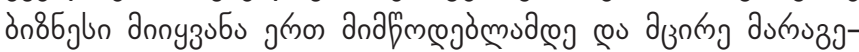

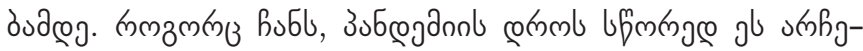

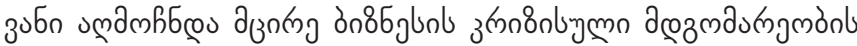

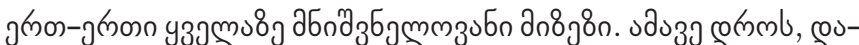

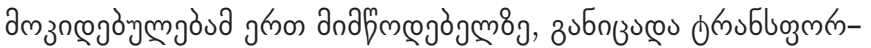

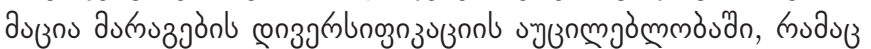

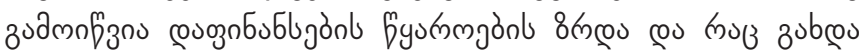

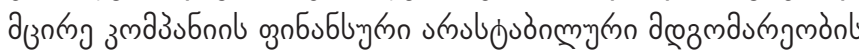
2n8̊g8n (Shengelia \& Gelashvili, 2020).

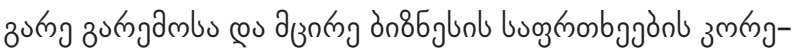

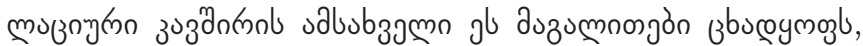

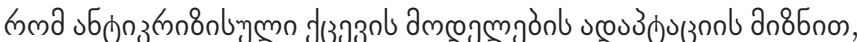

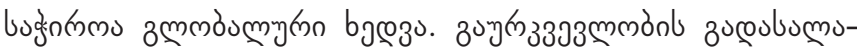

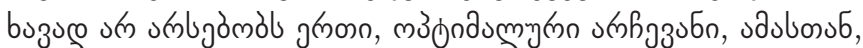

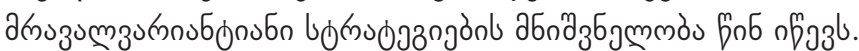

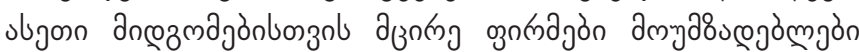

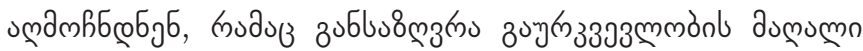

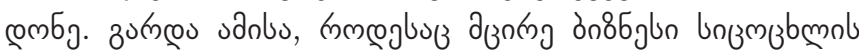

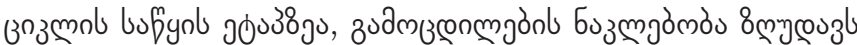
dol sкh

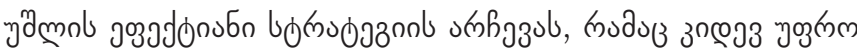

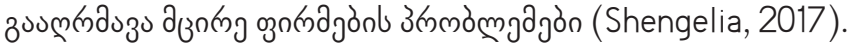

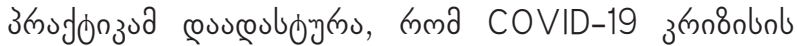

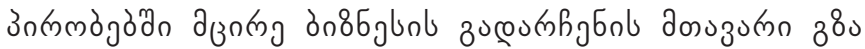
фаل

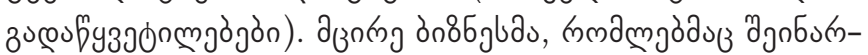

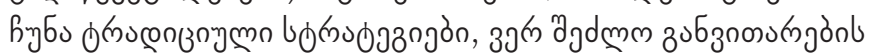

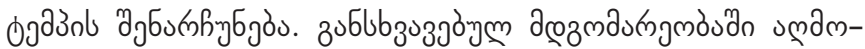

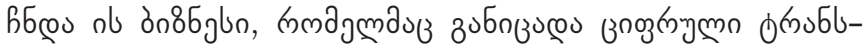

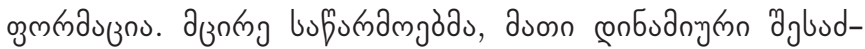

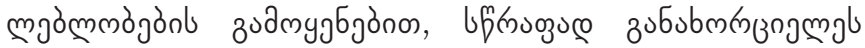

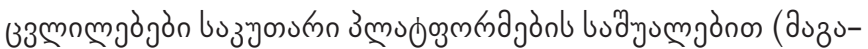

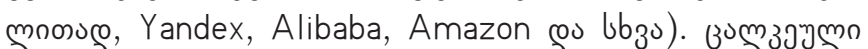

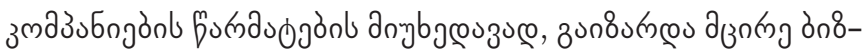

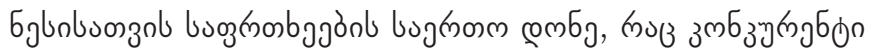

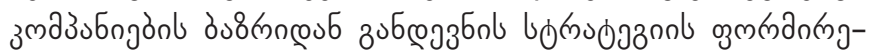
buan zuamobuoj.

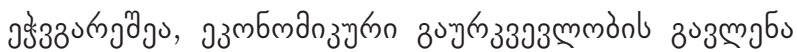

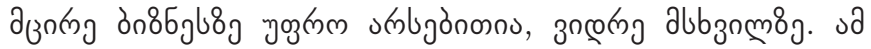

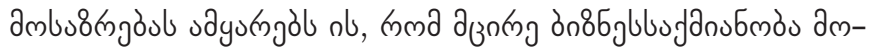

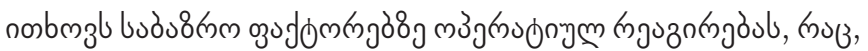

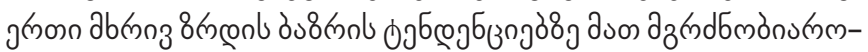

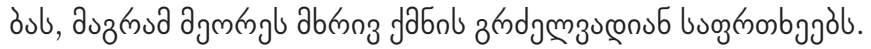

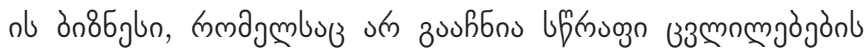

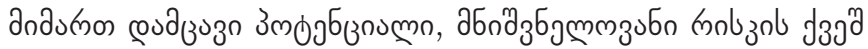
naymogjoso (Shengelia, 2014).

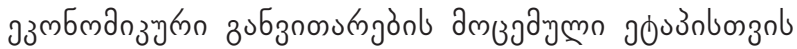

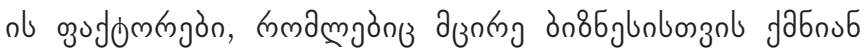

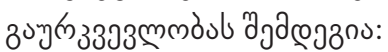

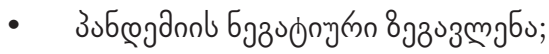

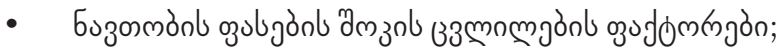

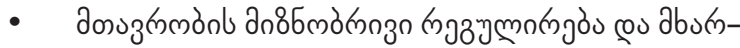
couggho;

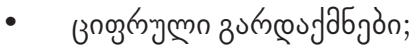

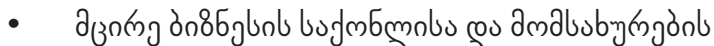
ambaumgonl u l

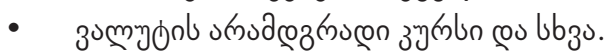

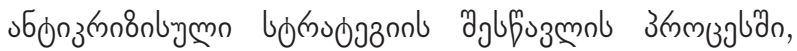

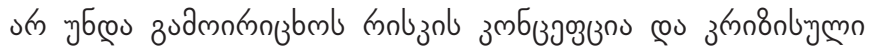

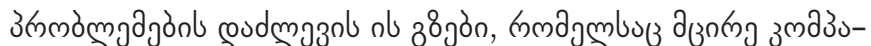

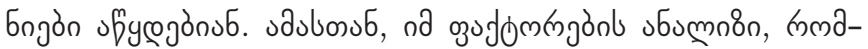

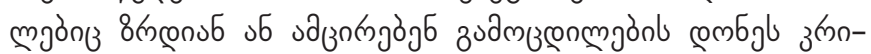

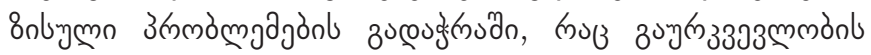

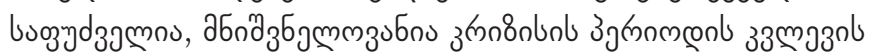

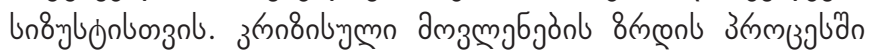

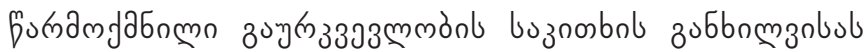

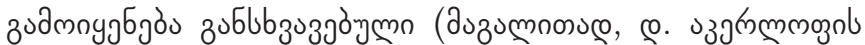

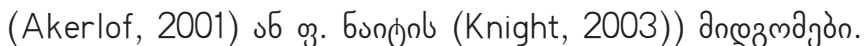

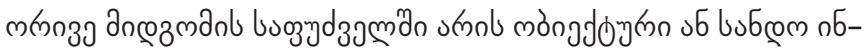




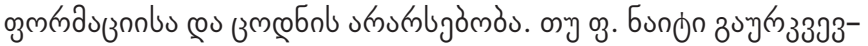

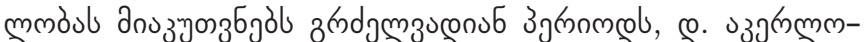

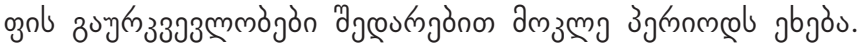

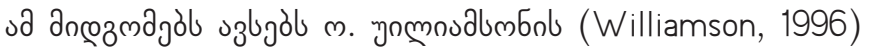

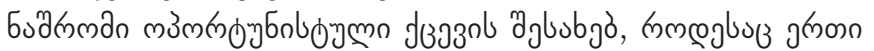

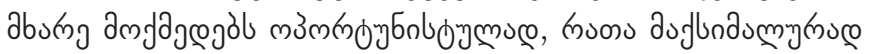

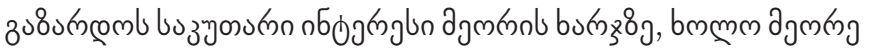

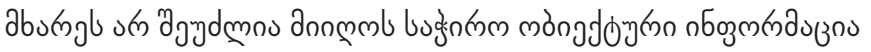

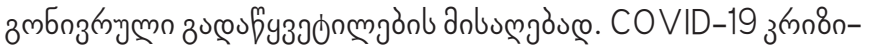

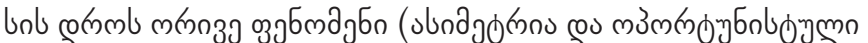

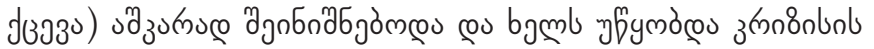

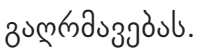

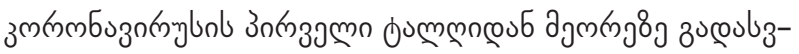

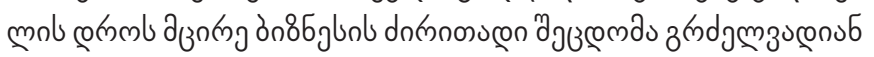

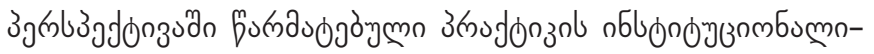

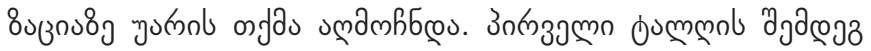
coग

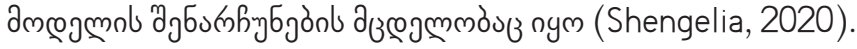

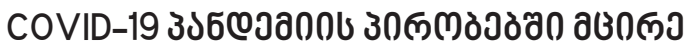

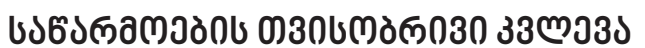

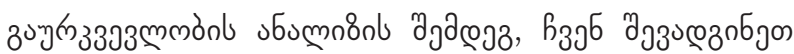

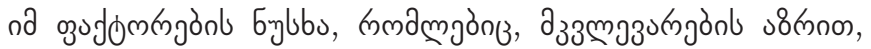

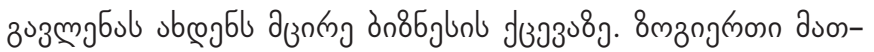

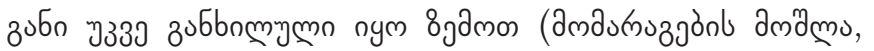

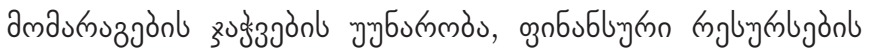

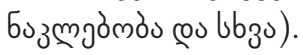

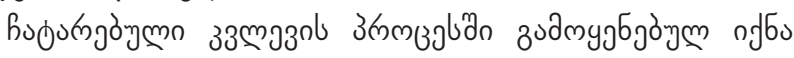

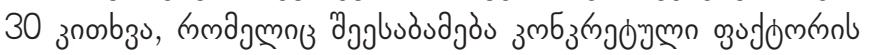

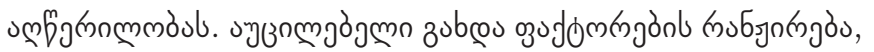

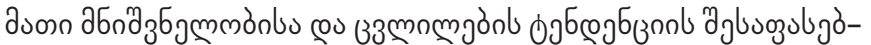

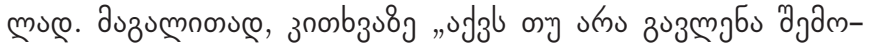

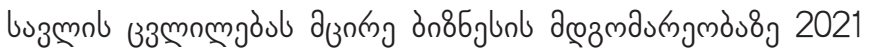

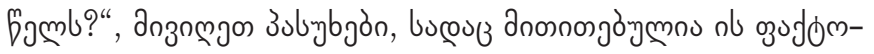

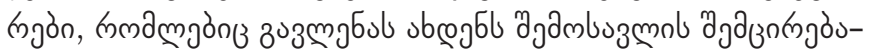

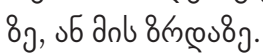

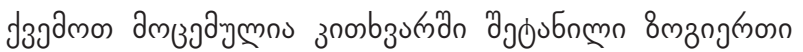

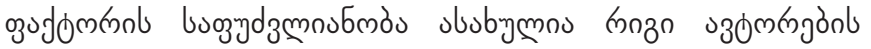

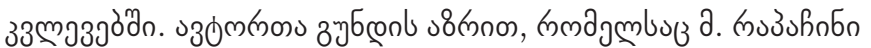

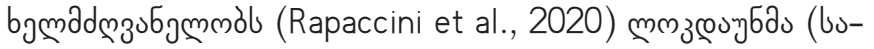

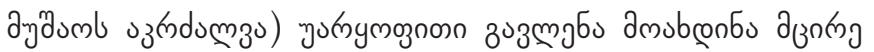

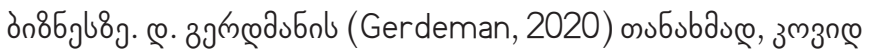

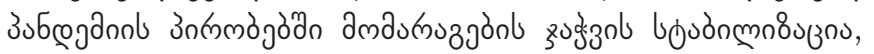

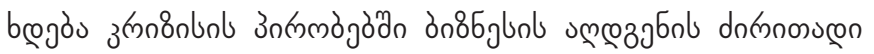
oु०Jomkn.

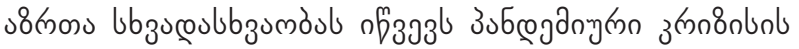

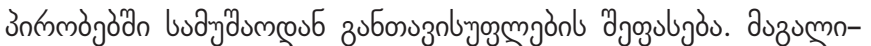

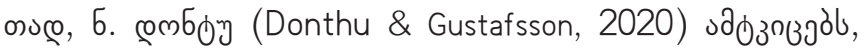

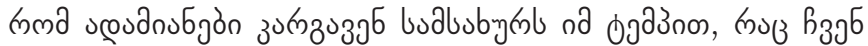

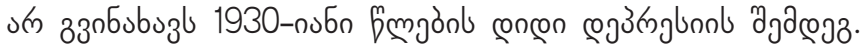
s.bumzin (Tarki et al., 2020) dnuh5ns, mma COVID-19

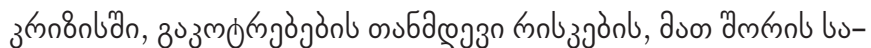

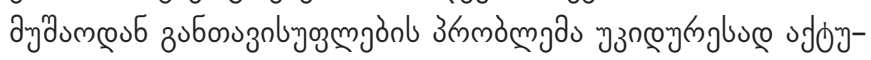
umymos.

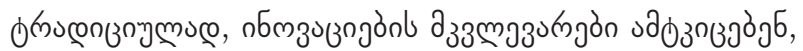

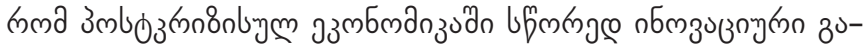

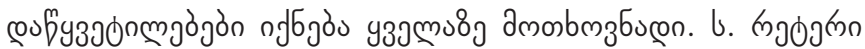

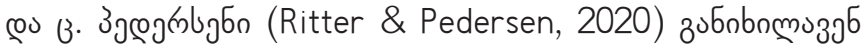

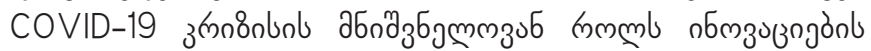

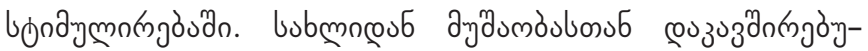

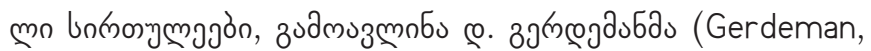

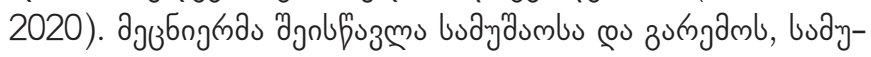

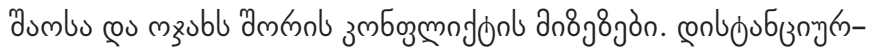

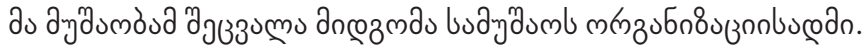

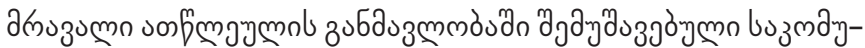

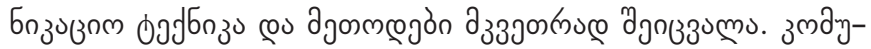

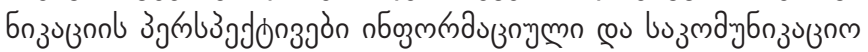

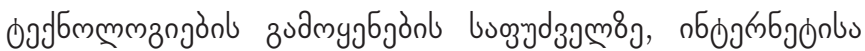

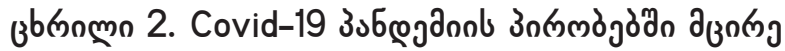

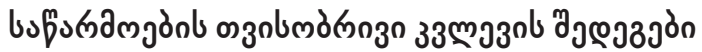

\begin{tabular}{|c|c|c|}
\hline oुगdom & 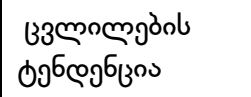 & $\%$ \\
\hline 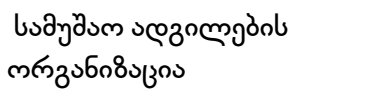 & 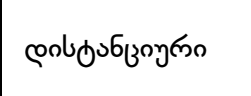 & 53,3 \\
\hline 3ucesbẹnby6umoubmòs & 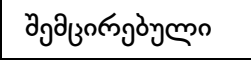 & 14,1 \\
\hline 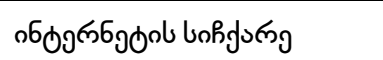 & smuluzasmonn & 29,4 \\
\hline 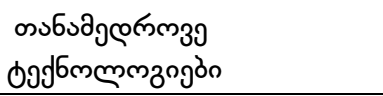 & smoluzasamoln & 6,9 \\
\hline 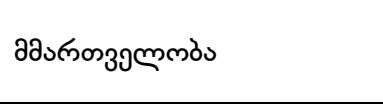 & 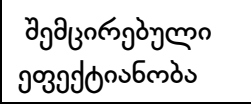 & 27,4 \\
\hline 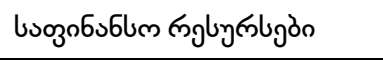 & smuluzasmonon & 51,9 \\
\hline 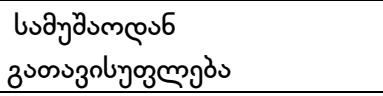 & 8mழฺ & 62,1 \\
\hline cubsbumfgòn & 8mp̧os & 32,9 \\
\hline 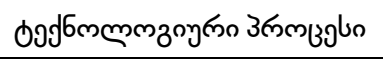 & 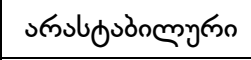 & 28,6 \\
\hline 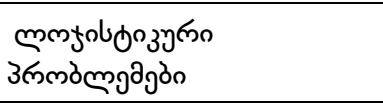 & bynmo & 41,9 \\
\hline yjamlsuzumo & 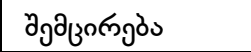 & 51,5 \\
\hline 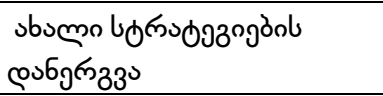 & 30ก8sm & 19,3 \\
\hline sbumo పglud mjàmmàjòn & 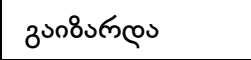 & 12,2 \\
\hline 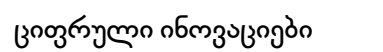 & 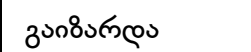 & 32,1 \\
\hline
\end{tabular}

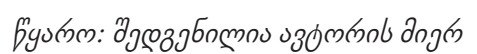




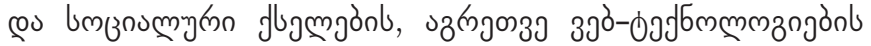

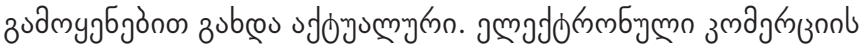

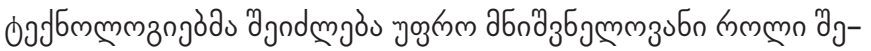

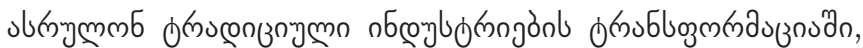

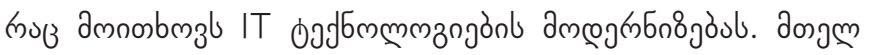

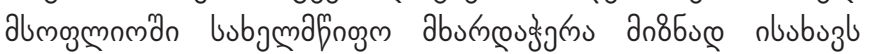

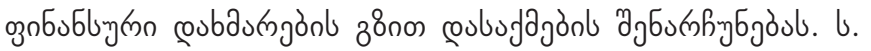

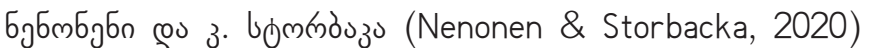

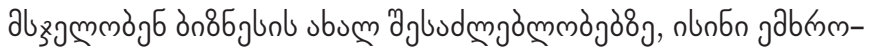

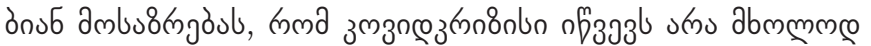

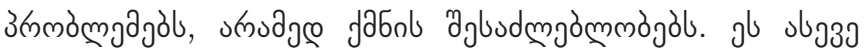
couboyn @ of

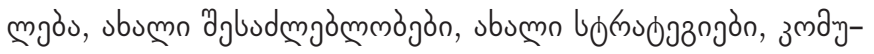

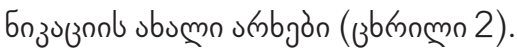

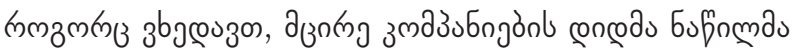

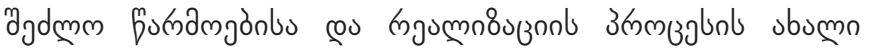

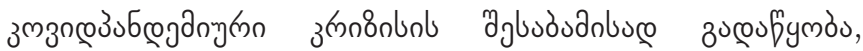

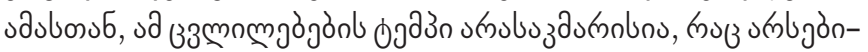

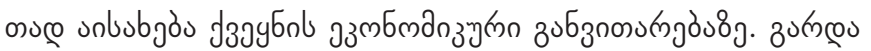

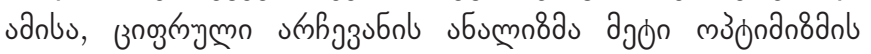

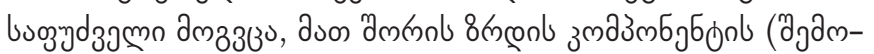

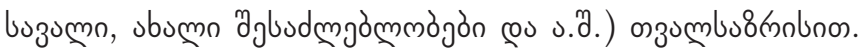

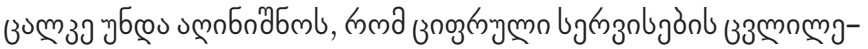

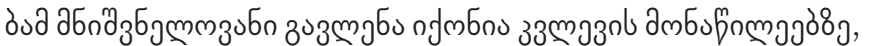

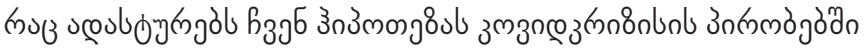

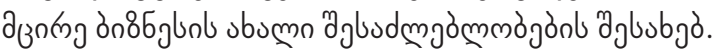

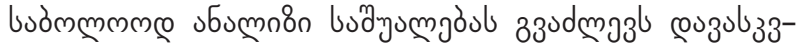

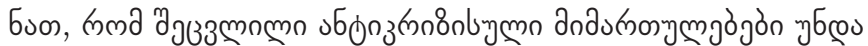

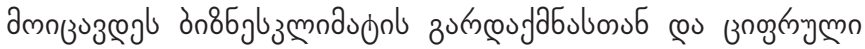

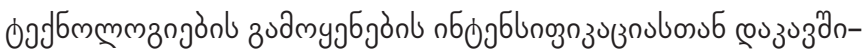

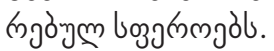

\section{esu336s}

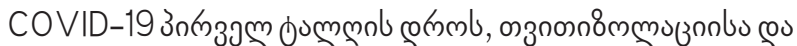

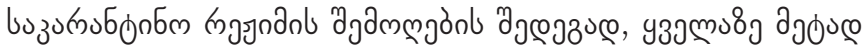

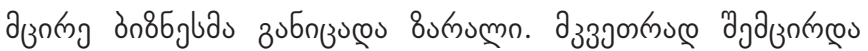

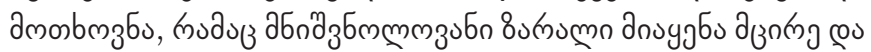

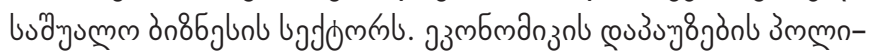

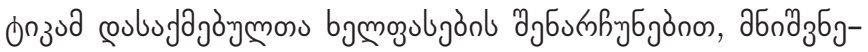

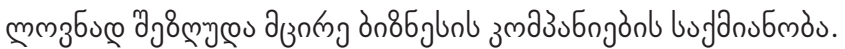

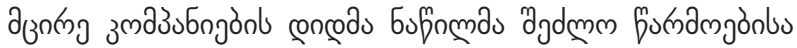

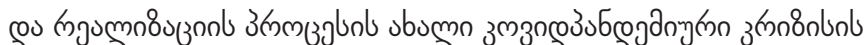

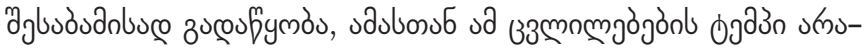

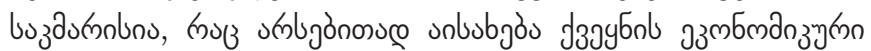

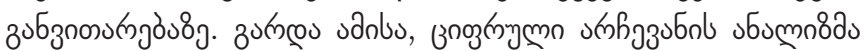

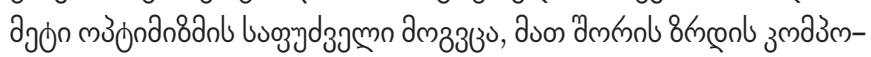

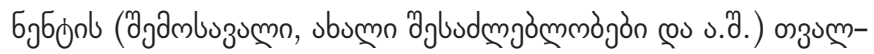

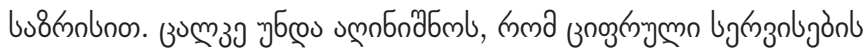

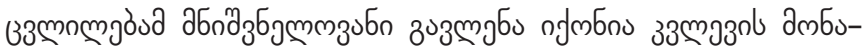

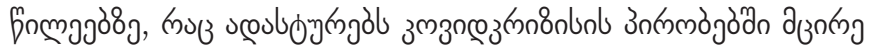

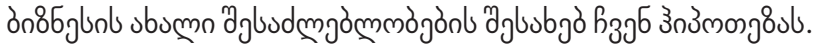

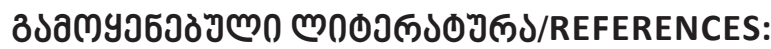

Adžić, S., \& Al-Mansour, J. (2021). Business analysisinthe times of COVID-19: Empirical testing of the contemporary academic findings// Management Science Letters, 11,1-10.

Akerlof, D. (2001). Identity and the Economics of Organizations. Journal of Economic Perspectives, 19(1), 9-32.

Berishvili, Kh. (2020). Implementation of the concept of business social responsibility in the political systems of social democracy. World economy and international economic relations. 3.

Carnevale, J. B., \& Hatak, I. (2020). Employeead justment and well-being in the era of COVID-19: Implications for human resource management. Journal of Business Research. 116, 183-187.

Donthu, N., \& Gustafsson, A., (2020). Effects of COVID-19 on business and research. Journal of Business Research. 117, $284-289$.

Gerdeman, D., (2020). How the Coronavirus Is Already Rewriting the Future of Business. HBS Working Knowledge, URL: http:// hbswk.hbs.edu/item/how-the-coronavirus-is-already- rewriting-the-future-of-business.

Haas R, 2020. The world: brief introduction. NY: Penguin Press.

Knight, Fr. (2003). Risk, Uncertainty, and Profit. NY: Penguin Press.

Kozak, Y., Shengelia, T., \& Gribincea, A. (2020). The current state of the world economy, international economic relations and the paradigm of global issues. World economy and international economic relations. 4, 5-11.

Nenonen, S., \& Storbacka, K., (2020). Don't adapt, shape! Use the crisis to shape your minimum viable system-And the wider market. Industrial Marketing Management, 2020, 88, 265-271.

OECD, (2020). Statistical Insights: Small, Medium andVulnerable. URL:http://www.oecd.org/sdd/business-stats/statisticalinsights-small-medium-and-vulnerable.htm

Rapaccini, M., Saccani, N., Kowalkowski C., Paiola M., \& Adrodegari, F., (2020). Navigating disruptive crises through service-ledgrowth: The impact of COVID-19 on Italian manufacturing firms. Industrial Marketing Management. 88, 225-237.

Shengelia, T. (2014). Tendencies of the Capital International Movement and Its Influence on the Economy of the Sovereign State. International Journal of Science, Technology and Medicine. 6 (1), 49. 
Shengelia, T. (2017). The role of state regulation of investment in the Georgia business development. International business in the world economic system.

Shengelia, T. (2017). The role of state regulation of investment in the Georgia business development. International business in the world economic system.

Shengelia, T., \& Gelashvil, M. (2020). Export potential of Georgia. World economy and international economic relations. 3, 29-33.

Shengelia, T., Gasviani, B. (2020). Establishment of the stages of organizational development in Georgian SME's. World economy and international economic relations. 3, 11-15.

Shengelia, T., Kirkitadze, Z. (2020). The Post-Coronavirus Economy of the World and Georgia. Economics and Business, 4.

Shengelia, T., Kozak, Y., \& Kirkitadze, Z. (2020). FDI motivation effects on Host countries. World economy and international economic relations. 4, 15-20.

Tarki, A., Levy, P., \& Weiss, J. (2020, March 20). The Coronavirus Crisis Doesn't Have to Lead to Layoffs. In Coronavirus and Business: The Insights You Need from Harvard Business Review. Harvard Business Review. https://www.harvard business. org/wp-content/uploads/2020/03/HBR_The_Coronavirus_Doesnt_Have_to_Lead_to_Layoffs.p

Williamson, Oliver E. (1996). The Economic Institutions of Capitalism. New York: Macmillan. 